\title{
NIH laboratory admits to fabricated embryo research, retracts paper
}

Washington. As retractions go, the one in the 29 May issue of Cell was short and unambiguous: data supporting a 1991 paper on mouse embryo development written by four researchers from the US National Institutes of Health (NIH) had "been fabricated by one of the authors ... without any knowledge by the others".

But cases of scientific misconduct these days are rarely that simple. Although there is no argument about what was done and who did it, the NIH case raises troubling questions about the ability of researchers in a laboratory to block a determined effort by a colleague to fabricate data.

The paper, entitled "Oct- 3 is a maternal factor required for the first mouse embryonic division" (Cell 64, 1103; 1991), came to the surprising and unexpected conclusion that embryonic cell division requires Oct-3, a protein that switches on genes, and that the protein may even regulate DNA replication in early development. This was more than anyone had expected of a humble transcription factor.

Oct-3, as it turns out, does nothing of the sort. When Louis Staudt and other researchers in his laboratory at NIH's National Cancer Institute (NCI) tried to replicate the results, they found that the key experiments no longer worked. Yet the only significant difference between their work and what had appeared in Cell was the people doing it. The first author was Mitchell Rosner, a graduate student who spent last year at NIH but who is now at the Harvard University Medical School.

When Rosner learned that Staudt could not replicate his findings, he volunteered to repeat the experiments. Staudt and other researchers in the laboratory set up a double blind test. Rosner prepared his reagents as usual, and when the codes were revealed the experiment again worked. But this time, when his colleagues examined the reagents, they discovered why.

Initially, contamination in the injected samples interfered with the embryonic development, a problem that had apparently disappeared after Rosner refined the reagents. In the subsequent investigation, the principal samples were shown to contain just what they should - antisense Oct-3 oligonucleotide to block Oct-3 in the developing embryos.

But the controls, rather than containing DNA without antisense Oct-3, turned out to be simply a buffer solution - essentially water. Rosner had 'solved' the contamination problem by removing the DNA entirely. In the replication trial he had apparently broken the code and, as he had in the previous experiments, surreptitiously switched test tubes for the control groups. No wonder, then, that the control embryos performed nearly as well as those that were not injected at all.

Confronted with his colleagues' suspicions, Rosner confessed. Staudt says that Rosner blamed the fabrication on "self-imposed pressure". Rosner did not return telephone calls last week.

Staudt reported the misconduct to officials at NCI and NIH. Philip Chen, the acting director of the $\mathrm{NIH}$ intramural programme, confirms that the case has been given to the NIH Office of Scientific

\section{“Recent Investigations have revealed that the experimental evidence supporting the conclusions of the paper by Rosner et al. has been fabricated by one of the authors (M.R.) without any knowledge by the others. We therefore retract this paper in its entirety. We sincerely apologize to anybody, within or outside the research community, who has been misled by this publication." - Cell.}

Integrity, which is in the midst of an extensive reorganization (see page 431).

Suzanne Rouffenbart, a spokeswoman for Harvard Medical School, says that Rosner told the school about the case and that the dean has assembled an advisory committee of faculty members. The committee is expected to submit a report later this month. Rosner, who was a candidate for a doctoral degree at Georgetown University Medical School when the paper appeared, has also withdrawn his candidacy for that degree.

Researchers familiar with the case say that it illustrates both the length to which a researcher may go to hide data fabrication and the difficulty of preventing such behaviour.

"If you didn't know it was a hoax, you'd say it was a fine piece of work, a wellcontrolled study," says Michael Green of the University of Massachusetts Medical Center. Confronting such deliberate fabrication, he says, "is frightening, but when the fright passes, you realize that it's virtually impossible to stop a person who is willing to act in a determined and malicious fashion."

By all accounts, the Rosner case did not fit either of two stereotypes of scientific misconduct: he was neither a junior researcher working alone and submitting data to a distracted laboratory chief nor an un- known student producing amazing results overnight for supervisors too pleased to examine the data closely.

Staudt says he worked closely with Rosner on the experiment and reviewed his progress daily. "I saw him put in the DNA tablets," he says. (Rosner apparently switched test tubes when he took the controls to part of the experiment in another room.)

In addition, the results were the product of long, hard work, and were built upon earlier efforts. Rosner was the lead author of a paper in Nature $(354,686 ; 1990)$ that Staudt says "took us a year to do and has been proven entirely correct and confirmed by other labs." Furthermore, Staudt says, Rosner was "working day and night".

To Alan Rabson, director of the Division of Cancer Biology, Diagnosis and Centers at $\mathrm{NCI}$, "this case points out that a truly determined person can deceive even a fulltime, conscientious scientist". To others, the episode proves that scientists can indeed police themselves. "This is a case of science working as it should," says Benjamin Lewin, the editor of Cell.

Staudt says that the case has taught him that "fraud is present and needs to be watched out for. I wish I had done the experiment again, with other people [preparing the reagents]. In the future, I'll be more ready to do that."

Others, however, doubt that anyone can deter an inventive researcher determined to fabricate data. They point out that replicating an experiment goes beyond repeating a single procedure and could require asking an outside researcher to recreate reagents that have taken months to produce. And they say that the extra effort is not justified.

"Even if you could find an independent person in your lab who would be willing to do the whole experiment again", says Green, "that would reduce your scientific productivity and increase the cost of the science by as much as a factor of two. The price you'd pay for that is not worth stopping the .001 per cent of fraudulent manuscripts."

The 15-month delay between publication and retraction was time enough for dozens of unsuspecting scientists to waste their time chasing the erroneous implications of the paper. Although that does not appear to have happened in this case, the potential damage from fraudulent science entering the literature has convinced Staudt that extra precautions, no matter how inconvenient, are needed to avoid another case of misconduct. That lesson, however, appears to be something that can be learned only at first hand.

Christopher Anderson 